\title{
A study on water film in saturated sand
}

\author{
LU X. B. ${ }^{1}$ and CUI P. ${ }^{2}$
}

\begin{abstract}
Water film can serve as a sliding surface and cause landslides on gentle slopes. The development of "water film" in saturated sand is analyzed numerically and theoretically based on a quasi-three-phase model. It is shown that stable water films initiate and grow if the choking state (where the fluid velocity decreases to near zero) remains steady in a liquefied sand column. Discontinuity can occur in pore water velocity, grain velocity and pore pressure after the initiation of a water film. However, the discontinuity and water film can disappear once the choking state is changed. The key to the formation of water film is the choking in the sand column caused by eroded fine grains.
\end{abstract}

Key Words: Saturated sand, Water film, Liquefaction

\section{Introduction}

The concept of "water film" in sand containing an impermeable layer was first suggested by Seed (1987) in his attempt to explain slope failures observed in earthquakes. This "water film" can serve as a sliding surface for post-liquefaction failure. This sliding surface can arouse landslides and debris flows on gentle slopes. The water film in saturated sand is a water gap due to non-uniform permeability of the sediment where the pore water is trapped by relatively low permeable layers. Sand grains do not support one another. They suspend under the condition of zero effective stresses (Scott, 1986), and eventually settle down because they have a higher density than water. The rate of settlement is restricted by the fact that water must flow upward around the sand grains (Bose and Dey, 2009). If liquefiable sand deposits are overlain by less permeable soils in a stratified deposit, the overlaying deposit can restrict the pore water from passing through. If there is no downward drainage through the deposit, this relative flow between the upward water flow and the settlement of grains at the interface, by continuity, must be equal to the velocity of settlement at the upper liquefied sand surface (Fiegel and Kutter, 1994). Thus, an accumulation of water in the form of a water gap forms at the interface. Feigel and Kutter (1994) and Malvick et al. (2008) performed centrifuge shake table tests to demonstrate the formation of water films in stratified sand. More recently, Kokusho (1999) performed shake table tests using sand samples containing a seam of non-plastic silt. Kokusho showed that water films formed beneath the silt layer. In this case, the column was subjected to horizontal dynamic loadings to simulate earthquakes. Experimental observations on the formation of water films in vertical columns of saturated sand contained in circular cylinders have also been reported by Zhang et al. (1999) and Peng et al. (2001). In both cases, care was taken in preparing the sample by feeding wetted uniform sand continuously into a column of water to avoid intentional stratification. However, small heterogeneity still existed due to nonuniform settlement velocity.

These researches revealed that liquefaction is a necessary condition for water film initiation and growth. In Zhang's experiment (1999), a sand column in a circular cylinder was subjected to a vertical impact. It

${ }^{1}$ Dr., Laboratory for Hydraulic and Ocean Engineering, Institute of Mechanics, Chinese Academy of Sciences, Beijing 100190, China, E-mail: xblu@imech.ac.cn

2 Prof., Key Laboratory for Mountain Hazard and Earth Surface Process, Institute of Mountain Hazard and Environment, Chinese Academy of Sciences, Chengdu 610041, China, E-mail: pengxcui@imde.ac.cn

Note: The original manuscript of this paper was received in Feb. 2009. The revised version was received in May 2010. Discussion open until Sept. 2011. 
was found that small heterogeneity of saturated sand may be aggravated during the settlement of sand after liquefaction. The water films occurred at the place where fine grains accumulated ( Fig. 1).

In the centrifuge experiments of Malvick et al. (2008), a stratified slope was born shaking. A thin layer of silt with a permeability of $3 \times 10^{-8} \mathrm{~m} / \mathrm{s}$ was embedded in the slope. The rest of the slope consisted of Nevada sand with a permeability of $5 \times 10^{-5} \mathrm{~m} / \mathrm{s}$. It was shown that the maximum increases of pore pressure and displacement occurred immediately under the silt clay (Fig. 2) after shaking. This indicates that the water film forming under the silt clay is caused by the net inflow of water in this zone due to the high pore pressure. The low permeability layer of silt clay plays an important role in the formation of water film.

In addition, Zheng et al. (2001), Lu et al. (2006) showed analytically that the water film had to be just beneath the fine sand layer. Malvick et al. (2006) discussed the development of water films using the concept of localization.

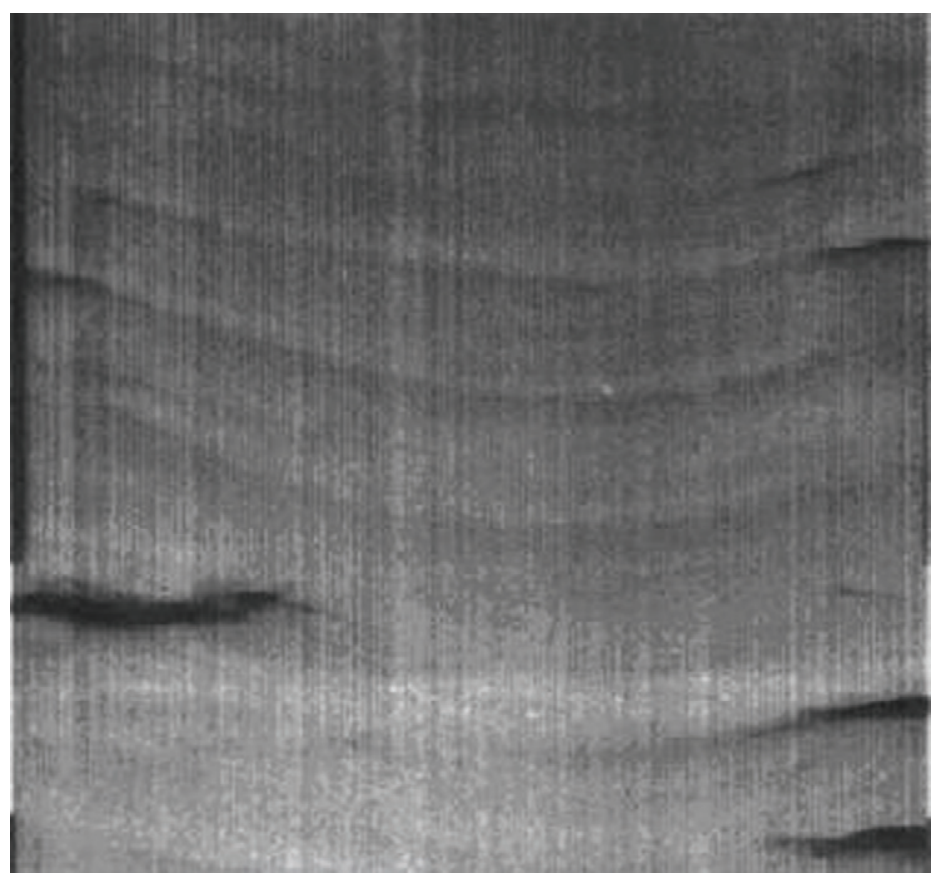

Fig. 1 Transverse water films in a sand column (Zhang et al., 1999)

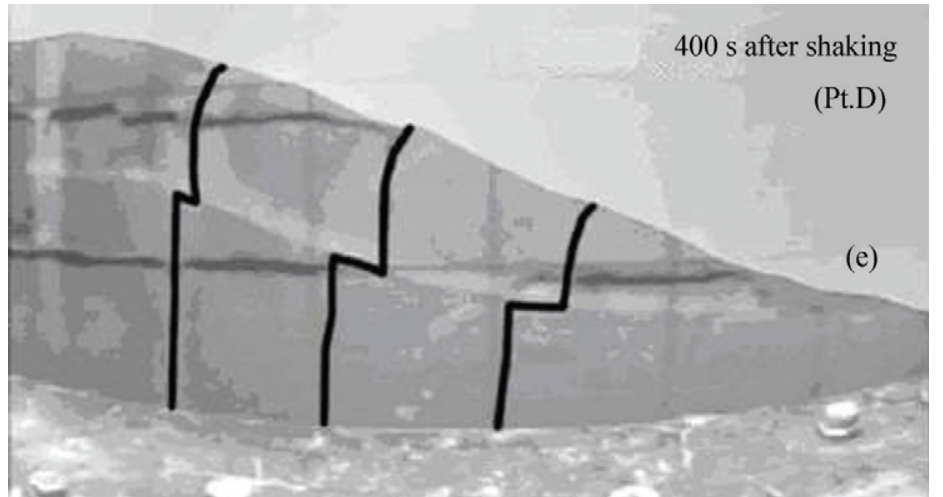

Fig. 2 Displacement and formation a water film after shaking (Malvick et al., 2006)

The formation mechanism of water films in stratified sand, especially in sand with porosity distributed continuously, is a process that has not been researched extensively. This paper reports the further analysis 
of the formation mechanisms of water films on the basis of the above mentioned works.

In this paper, a quasi-three-phase model is presented to describe the movement of liquefied sand. Although a full description is not available, a simple empirical model will be devised to explain qualitatively the main features observed experimentally. Then, theoretical analyses and numerical simulations will be used to understand the mechanism of water films.

\section{Formulation of the problem}

Figure 3 shows a horizontal sand stratum, which is water saturated with porosity and other parameters changing only vertically. The $\mathrm{x}$ axis is upward (Fig. 3).

A set of simplified quasi-three-phase flow equations is presented in the following, under the assumptions that (1) the flow is one dimensional; (2) the inertia effect may be neglected; (3) only the simplest form of interaction between water and grains is considered and; (4) the whole sand column is liquefied at the beginning. A broad grain size distribution means that some of the fine grains may be washed away to become part of the percolating fluid or re-deposited later somewhere down stream (Alekseevskiy et al., 2008). This may turn an initially homogeneous sand column into an inhomogeneous sand column. The heterogeneity can aggravate with time and flow rate. The eroded grain mass is assumed to proportional to the relative velocity between water and grains but limited by the mass of fine grains in pores. Hence, the problem lies in properly describing the transport of these fine grains and its effect on permeability.

\subsection{Erosion relation}

In experiments the water films formed only when the grain size distribution was broad and contained fine grains. These experiments suggest that the fine grains should be flushed away by the percolating water. This results in the change of initial porosity and the turbidity of percolating water. Changes in initial porosity and turbidity both alter the permeability.

Fine grain mass transferred to water is assumed to be proportional to the relative velocity between grains and water, but inversely proportional to the fine grain mass in the percolating fluid. There is a limit to the amount of fine grains that can be transported (Fazli et al., 2008; Wang et al., 2008; Ghodsian and Vaghefi, 2009; Yu et al., 2009). Thus, the erosion relation is (Cheng et al., 2000):

$$
\begin{gathered}
\frac{1}{\rho_{s}}\left(\frac{\partial Q}{\partial t}+u_{s} \frac{\partial Q}{\partial x}\right)=\frac{1}{T}\left(\frac{u-u_{s}}{u^{*}}-q\right) \text { if }-\varepsilon(x, 0) \leq \frac{Q}{\rho_{s}} \leq \frac{Q_{c}(x)}{\rho_{s}} \\
\frac{1}{\rho_{s}}\left(\frac{\partial Q}{\partial t}+u_{s} \frac{\partial Q}{\partial x}\right) \leq 0 \quad \text { otherwise }
\end{gathered}
$$

in which the first term $\frac{u-u_{s}}{u^{*}}$ on the right side of the first equation shows how the fine grains are transferred to water, the second term $-q$ describing deposition places a limit on the amount of fine grains that can be carried in the percolating fluid, $q$ is the ratio of the volume of fine grains to porosity, $Q$ is the fine grain mass eroded per unit volume of the sand/water mixture, $\rho_{s}$ is the density of the grains, $u$ is the velocity of percolating fluid containing fine sand grains, $u_{s}$ is the velocity of sand grains, $T$ and $u^{*}$ are the characteristic time and velocity in this problem, respectively, $\varepsilon(x, t)$ is the porosity, $Q_{c}(x)$ is the maximum of $Q$ that can be eroded at $\mathrm{x}$.

\subsection{Conservation equations}

Considering the erosion of fine grains, the pore is filled by two parts: one is the fine sand eroded from the skeleton $q$, and the second is pure water $\varepsilon-q$. Assuming that fine grains flow with the pore water, the mass conservation equations can be described by (Cheng et al., 2000)

$$
\begin{gathered}
\frac{\partial(\varepsilon-q) \rho}{\partial t}+\frac{\partial(\varepsilon-q) \rho u}{\partial x}=0 \\
\frac{\partial q \rho_{s}}{\partial t}+\frac{\partial q \rho_{s} u}{\partial x}=G=\frac{\partial Q}{\partial t}+u_{s} \frac{\partial Q}{\partial x} \\
\frac{\partial(1-\varepsilon) \rho_{s}}{\partial t}+\frac{\partial(1-\varepsilon) \rho_{s} u_{s}}{\partial x}=-G=-\frac{\partial Q}{\partial t}-u_{s} \frac{\partial Q}{\partial x}
\end{gathered}
$$


in which $\rho$ is the density of water, $G$ is the erosion-rate, e.g., the mass of fine grains eroded from the skeleton in unit time.

Combining Eqs. (3)-(5) yields

$$
\varepsilon u+(1-\varepsilon) u_{s}=U(t)
$$

in which $U(t)$ is the flow rate of water per unit cross sectional area of the sand column.

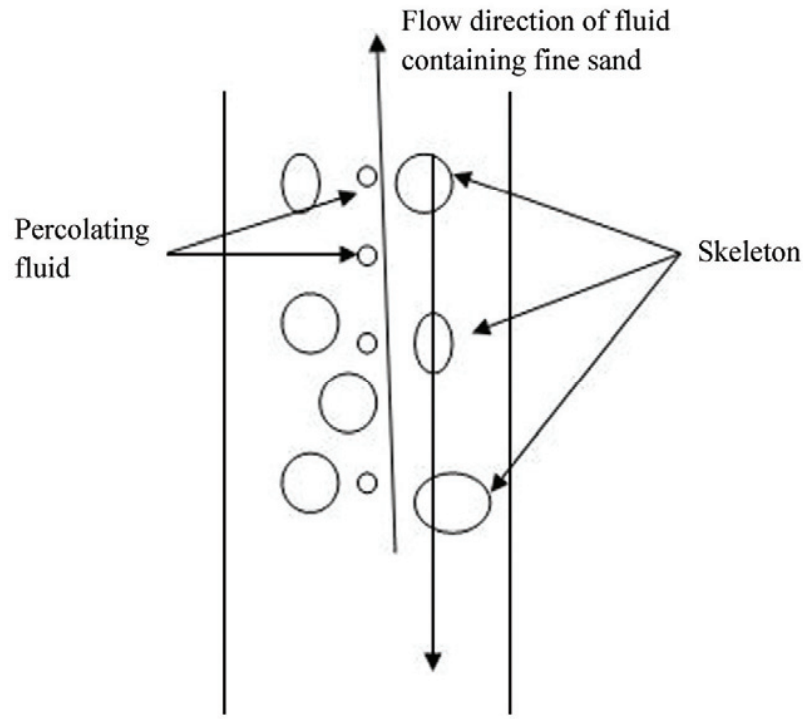

The move direction of gains

Fig. 3 Sketch of the model (A sand column with parameters only vertically changeable is considered. The fine grains eroded from the skeleton move upwards with pore water flow while the coarse grains settle downwards. )

Considering the momentum transferred by the fine sand eroded from the skeleton, the momentum conservation equations are adopted as:

$$
\begin{gathered}
{\left[(\varepsilon-q) \rho+q \rho_{s}\right]\left(\frac{\partial u}{\partial t}+u \frac{\partial u}{\partial x}\right)=-\varepsilon \frac{\partial p}{\partial x}-\frac{\varepsilon^{2}\left(u-u_{s}\right)}{k(\varepsilon, q)}-} \\
{\left[(\varepsilon-q) \rho+q \rho_{s}\right] g} \\
{\left[(\varepsilon-q) \rho+q \rho_{s}\right]\left(\frac{\partial u}{\partial t}+u \frac{\partial u}{\partial x}\right)+(1-\varepsilon) \rho_{s}\left(\frac{\partial u_{s}}{\partial t}+u_{s} \frac{\partial u_{s}}{\partial x}\right)=-\frac{\partial p}{\partial x}-\frac{\partial \sigma_{e}}{\partial x}-} \\
{\left[(\varepsilon-q) \rho+q \rho_{s}\right] g-(1-\varepsilon) \rho_{s} g-\left(\frac{\partial Q}{\partial t}+u_{s} \frac{\partial Q}{\partial x}\right)\left(u-u_{s}\right)}
\end{gathered}
$$

in which Eq. (7) is for percolating fluid and Eq. (8) for the total momentum. The last term on the right side of Eq. (8) is the momentum of eroded fine grains. In Eqs. (7) and (8), $p$ is the pore pressure, $k$ is the physical permeability $\left(k=K / \rho_{w} g, \rho_{w}\right.$ is the density of the water, $K$ is the Darcy's permeability, and $g$ is the gravity acceleration), and $\sigma_{e}$ is the effective stress.

Obviously, $k$ increases positively with increasing $\varepsilon$ and decreasing $q$. For simplicity without loss of generality, $k$ is assumed to be proportional to $\varepsilon$ and inversely proportional to $q$ (Lu et al., 2006):

$$
k(\varepsilon, q)=k_{0} f(q, \varepsilon)=k_{0}(-\alpha q+\beta \varepsilon)
$$

in which $\alpha, \beta$ are parameters and $1<\beta<<\alpha$. Since we think the choking state is mainly caused by the eroded fine grains, $\alpha$ is chosen to be much greater than $\beta$, in order to guarantee changes in $q$ have a greater influence than that of $\varepsilon$.

In experiments, the water film sometimes forms and expands or even disappears. This may be caused by 
the formation or disappearance of choked states in the sand layer. Thus, two assumptions are adopted in the following: (1) once the choking occurs, i.e., the permeability $k$ drops to zero at any place, the choking state remains unchanged; and (2) The choking state may disappear once the gradient of pore pressure is over a critical value $p_{c r}$.

2.3 Discontinuity relations and the conditions for formation of water films

The expansion of water films depends on the discontinuity. Assuming the velocity of discontinuity is $w$, and using transformation of coordinate $\xi=x-w t$, which is often adopted in the analysis of discontinuity, e.g. shock wave (Lu et al., 2006), Eqs. (4) (8) become

$$
\left\{\begin{array}{c}
\frac{d}{d \xi}(\varepsilon-q)(u-w)=0 \\
\frac{d}{d \xi} q(u-w)=G \\
\frac{d}{d \xi}(1-\varepsilon)\left(u_{s}-w\right)=-G \\
\frac{d}{d \xi}\left[(\varepsilon-q) \rho+q \rho_{s}\right] u(u-w)+\frac{d}{d \xi}(1-\varepsilon) \rho_{s} u_{s}\left(u_{s}-w\right)+\frac{d}{d \xi}\left(p+\sigma_{e}\right)= \\
-\left[(\varepsilon-q) \rho+(1-\varepsilon+q) \rho_{s}\right] \\
\frac{1}{\varepsilon} \frac{d}{d \xi}\left[(\varepsilon-q) \rho+q \rho_{s}\right] u(u-w)+\frac{d}{d \xi} p=-\frac{H}{\varepsilon}-\left[\rho+\frac{q}{\varepsilon}\left(\rho_{s}-\rho\right)\right]
\end{array}\right.
$$

where $H$ is the interaction assumed to be proportional to the velocity difference between pore water and grains and $H=n^{2}\left(u-u_{s}\right) / k$ (Lu et al., 2004), $\sigma_{e}$ is the effective stress.

Then, five discontinuity relations may be obtained by integrating Eq. (10) along the discontinuity:

$$
\left\{\begin{array}{c}
\rho_{u}^{+}=\rho^{-} \\
\left(\varepsilon^{+}-q^{+}\right)\left(u^{+}-w^{+}\right)=\left(\varepsilon^{-}-q^{-}\right)\left(u^{-}-w^{-}\right) \\
\left(1-\varepsilon^{+}\right)\left(u_{s}^{+}-w^{+}\right)=\left(1-\varepsilon^{-}\right)\left(u_{s}^{-}-w^{-}\right) \\
\varepsilon^{+} \rho_{u}^{+} u^{+}\left(u^{+}-w^{+}\right)+\left(1-\varepsilon^{+}\right) \rho_{s}^{+} u_{s}^{+}\left(u_{s}^{+}-w^{+}\right)+p^{+}+\sigma_{e}^{+}= \\
\varepsilon^{-} \rho_{u}^{-} u^{-}\left(u^{-}-w^{-}\right)+\left(1-\varepsilon^{-}\right) \rho_{s}^{-} u_{s}^{-}\left(u_{s}^{-}-w^{-}\right)+p^{-}+\sigma_{e}^{-} \\
\frac{1}{2} \rho_{u}^{+}\left(u^{+}-w^{+}\right)^{2}+p^{+}=\frac{1}{2} \rho_{u}^{-}\left(u^{-}-w^{-}\right)^{2}+p^{-}
\end{array}\right.
$$

in which $\rho_{u}=\rho+\frac{q}{\varepsilon}\left(\rho_{s}-\rho\right)$, assuming that $q / \varepsilon$ and $\rho_{u}$ are constant at the discontinuity. In the water film ( $\varepsilon=1)$, the water velocity is $u_{1}$, the pore pressure is $p_{1}$. Outside the water film $(\varepsilon \neq 1)$, the parameters are the porosity, the water velocity, pore pressure, grain velocity and effective stress $\varepsilon, u, p, u_{s}, \sigma_{e}$. Let $u-u_{1}=U, w-u_{1}=W$, with $W$ being the velocity of discontinuity relative to the water film.

Then derive the following relations

$$
\left\{\begin{array}{c}
u_{s}=w \text { or } u_{s}-u_{1}=W \\
\varepsilon U+(1-\varepsilon) W=0 \\
\Delta p=p_{1}-p=\frac{1}{2} \rho_{u}\left[(U-W)^{2}-W^{2}\right]=\frac{1}{2} \frac{1-\varepsilon^{2}}{\varepsilon^{2}} \rho_{u} W \\
\Delta p-\sigma_{e}=-\rho_{u} W U=\frac{1-\varepsilon}{\varepsilon} \rho_{u} W^{2} \\
\sigma_{e}=\frac{1}{2} \rho_{u} U^{2}=\frac{1}{2} \frac{(1-\varepsilon)^{2}}{\varepsilon^{2}} \rho_{u} W^{2}
\end{array}\right.
$$

It is clear that $W$ and $U$ will both vanish when $\sigma_{e}$ is zero, meaning that there is no water film in this case. The persistence or expansion of water films requires either nonzero effective stress or other 
conditions such as choking in sand.

\section{Initiation of water films}

Based on the model above, water film develops in a liquefied sand column (the effective stress and the shear stress are both zero), where coarse grains settle downwards and water with fine grains eroding from the skeleton move upwards.

Consider the fixed boundary, i.e., $u$ and $u_{s}$ are both zero at $x=0$, then the total mass flow rate from Eq. (6) is:

$$
\varepsilon u+(1-\varepsilon) u_{s}=U(t)=0
$$

Using Eqs. (1), (2), (9) and (10), Eqs. (4), (5), (7) and (8) are reduced to

$$
\left\{\begin{array}{c}
\frac{\partial \varepsilon}{\partial t}+\frac{\partial u \varepsilon}{\partial x}=\frac{1}{T}\left[\frac{u}{u *(1-\varepsilon)}-q\right] \\
\frac{\partial q}{\partial t}+\frac{\partial u q}{\partial x}=\frac{1}{T}\left[\frac{u}{u *(1-\varepsilon)}-q\right] \\
\frac{1-\varepsilon}{\varepsilon}\left[(\varepsilon-q) \frac{\rho}{\rho_{s}}+q\right]\left(\frac{\partial u}{\partial t}+u \frac{\partial u}{\partial x}\right)+\varepsilon\left(\frac{\partial u}{\partial t}-u \frac{\varepsilon}{1-\varepsilon} \frac{\partial u}{\partial x}\right)+\frac{u}{1-\varepsilon}\left(\frac{\partial \varepsilon}{\partial t}-u \frac{\varepsilon}{1-\varepsilon} \frac{\partial \varepsilon}{\partial x}\right) \\
=-\frac{\varepsilon u}{1-\varepsilon} \frac{1}{k_{0} \rho_{s} f(q, \varepsilon)}-\frac{u}{\varepsilon(1-\varepsilon)}\left(\frac{u}{T u *(1-\varepsilon)}-q\right)+\frac{1-\varepsilon}{\varepsilon}(\varepsilon-q)\left(1-\frac{\rho}{\rho_{s}}\right) g
\end{array}\right.
$$

It is observed in experiments that water film formation is so slow that the inertia effect can be neglected, thus Eq. (14) can be further simplified under the condition of erosion/re-deposition. It is reasonable to take $T$ as the appropriate characteristic time. And let $u_{t}$ denote the characteristic velocity and let $L$ denote the characteristic length of the problem, then Eq. (14) can be written in a non-dimensional form in terms of

$$
\bar{u}=\frac{u}{u_{t}}, \tau=\frac{t}{T}, \xi=\frac{x}{L}
$$

The first two equations in Eq. (14) become

$$
\left\{\begin{array}{l}
\frac{\partial \varepsilon}{\partial \tau}+\frac{T u_{t}}{L} \frac{\partial \varepsilon \bar{u}}{\partial \xi}=\bar{u} \frac{u_{t}}{u^{*}(1-\varepsilon)}-q \\
\frac{\partial q}{\partial \tau}+\frac{T u_{t}}{L} \frac{\partial q \bar{u}}{\partial \xi}=\bar{u} \frac{u_{t}}{u^{*}(1-\varepsilon)}-q
\end{array}\right.
$$

For $T g / u_{t} \gg$, i.e., the gravity effect dominates and the inertia terms are negligible, the last equation of Eq. (14) becomes

when $u_{t}$ is

$$
\bar{u}=\left(\frac{1-\varepsilon}{\varepsilon}\right)^{2}(\varepsilon-q) f(q, \varepsilon) \frac{k_{0} \rho_{s} g\left(1-\rho / \rho_{s}\right)}{u_{t}}=\left(\frac{1-\varepsilon}{\varepsilon}\right)^{2}(\varepsilon-q) f(q, \varepsilon)
$$

$$
u_{t}=k_{0} \rho_{s} g\left(1-\rho / \rho_{s}\right)
$$

Thus, up to a factor near unit, $u_{t} /(1-\varepsilon)$ is the settlement velocity of grains in a uniform sand column with constant permeability coefficient $k_{0}$. The problem now reduces to finding $\varepsilon(\xi, \tau)$. and $q(\xi, \tau)$ as a solution to Eq. (16). The initial conditions are:

$$
\varepsilon(\xi, 0)=\varepsilon_{0}(\xi), q(\xi, 0)=0
$$

In order to avoid complication due to consolidation wave (Scotter, 1986) from the bottom of the sand column, the sand column is assumed long enough for water film development before the wave arrives.

Then rewrite Eq. (16) as:

$$
\begin{aligned}
& \frac{\partial \varepsilon}{\partial \tau}+\frac{\partial \overline{u \varepsilon}}{\partial \zeta}=\bar{u} \frac{u_{t}}{u^{*}(1-\varepsilon)}-q \\
& \frac{\partial q}{\partial \tau}+\frac{\partial \overline{u q}}{\partial \varsigma}=\bar{u} \frac{u_{t}}{u^{*}(1-\varepsilon)}-q
\end{aligned}
$$

which can be shown to be hyperbolic with the characteristic equations as 


$$
\begin{aligned}
\text { The } \mathrm{C}^{-} \text {characteristic } & \frac{\mathrm{d} \zeta}{\mathrm{d} \tau}=\bar{u}+\varepsilon \frac{\partial \bar{u}}{\partial \varepsilon}+q \frac{\partial \bar{u}}{\partial q} \\
\text { The } \mathrm{C}^{+} \text {characteristic } & \frac{\mathrm{d} \zeta}{\mathrm{d} \tau}=\bar{u}
\end{aligned}
$$

Along the $\mathrm{C}^{-}$and $\mathrm{C}^{+}$characteristics, $\varepsilon$ and $q$ satisfy

$$
\begin{aligned}
& \frac{d \varepsilon}{d \tau}+\frac{\frac{\partial \bar{u}}{\partial q}}{\frac{\partial \bar{u}}{\partial \varepsilon}} \frac{d q}{d \tau}=\left(1+\frac{\frac{\partial \bar{u}}{\partial q}}{\partial \bar{u}}\right)\left(\frac{\lambda}{1-\varepsilon} \bar{u}-q\right) \\
& \frac{d \varepsilon}{d \tau}-\frac{\varepsilon}{q} \frac{d q}{d \tau}=\left(1-\frac{\varepsilon}{q}\right)\left(\frac{\lambda}{1-\varepsilon} \bar{u}-q\right)
\end{aligned}
$$

Then the solution is completely determined when the values of $\varepsilon$ and $q$ are prescribed at $t=0$ for $-\infty<\zeta<\infty$.

As for the initiation conditions of water films, consider first the magnitude of the parameter $u_{t} T / L$. In this equation, $T$ is time for settlement, $u_{t}$ is the settlement velocity of sand grains and $L$ is the interval between two water films. In the experiments of Zhang et al. (1999) and Peng et al. (2001), $T \sim 20 \mathrm{~s}$ and $u_{t}=10^{-4} \sim 10^{-5} \mathrm{~m} / \mathrm{s}$, while $L$ is $0.06 \mathrm{~m}$. Hence $u_{t} T / L$ ranges between $0.03 \sim 0.003$. Therefore, when the initial non-uniformity of the sand column is small $(\delta<<1)$, the second term in Eq. (20) can be neglected until the non-uniformity becomes sufficiently large and concentrated at certain locations. Consequently, for a limited period of time Eq. (20) can be further simplified to

$$
\begin{aligned}
\frac{\partial \varepsilon}{\partial \tau} & =\bar{u} \frac{u_{t}}{u *(1-\varepsilon)}-q \\
\frac{\partial q}{\partial \tau} & =\bar{u} \frac{u_{t}}{u^{*}(1-\varepsilon)}-q
\end{aligned}
$$

which shows that $\varepsilon$ and $\tau$ are periodic in $\varsigma$ when $\varepsilon_{0}(\eta)$ is also periodic. Taking into account quadrature, Eq. (17) yields

$$
\tau=\int_{0}^{q} \frac{\varepsilon=\varepsilon_{0}(\varsigma)+q}{\frac{u_{t}}{u_{\bullet}} \frac{1-\varepsilon_{0}(\varsigma)-q}{\left[\varepsilon_{0}(\varsigma)+q\right]^{2}} \varepsilon_{0}(\varsigma)\left(\alpha q+\alpha \varepsilon_{0}(\varsigma)-\beta q\right)-q}
$$

This indicates that water films are likely to develop when $q$ reaches the largest value in the shortest time. In particular, it shows that water films would develop at equal intervals of $x$ if the initial porosity distribution is periodic in $x$. However, this solution is not sufficiently accurate in describing how water film expands because the non-linear terms in Eq. (20) will be no longer negligible.

In the above discussions a number of constants are involved, say, $\alpha, \beta$ and $\lambda$, are in dimensionless form. In the following, the constraints will be put on these constants to ensure the development of water films. Consider the case where the initial porosity at distances far greater than $T u_{t}$ from $\varsigma=0$ is constant, then

$$
\varepsilon_{0}(\varsigma) \rightarrow \varepsilon_{0}^{+} \text {as } \varsigma \rightarrow \infty \text { and } \varepsilon_{0}(\varsigma) \rightarrow \varepsilon_{0}^{-} \text {as } \varsigma \rightarrow-\infty
$$

Water film may develop when the two parts of the sand column separate and this depends on the grain velocity $u_{s}$ at large $|\varsigma|$ as $\tau \rightarrow \infty$. According to Eq. (25), $\tau \rightarrow \infty$ requires the denominator in the integrand to be zero. This allows solving for porosity $\varepsilon^{+}$and $\varepsilon^{-}$at $\varsigma \rightarrow \pm \infty$ from the following equations as $\tau$ approaches infinity

$$
\varepsilon_{0}^{ \pm}\left[1+\lambda \frac{1-\varepsilon^{ \pm}}{\left(\varepsilon^{ \pm}\right)^{2}}\left(\alpha \varepsilon^{ \pm}+\beta \varepsilon_{0}^{ \pm}-\beta \varepsilon^{ \pm}\right)\right]-\varepsilon^{ \pm}=0
$$

The corresponding grain velocity is 


$$
u_{s}^{ \pm}=\frac{\varepsilon_{0}^{ \pm}}{\varepsilon^{ \pm}}\left(1-\varepsilon^{ \pm}\right)\left[(\beta-\alpha)\left(\varepsilon^{ \pm}-\varepsilon_{0}^{ \pm}\right)-\alpha \varepsilon_{0}^{ \pm}\right]
$$

This places a constraint on $\alpha, \beta, \lambda$ at given $\varepsilon_{0}^{+}$and $\varepsilon_{0}^{-}$. As an example, taking $\varepsilon_{0}^{-}=0.408$, $\varepsilon_{0}^{+}=0.392, \quad \alpha=1, \quad \beta=56$ and $\lambda=14$, it yields $u_{s}^{+}-u_{s}^{-}=3.56 \times 10^{-3} \quad$ as $\tau \rightarrow \infty$ with $u_{s 0}^{+}-u_{s 0}^{-}=3.2 \times 10^{-3}$ as $\tau \rightarrow 0$.

It follows that under the present condition one or several water films will eventually develop near $\varsigma=0$.

\section{Numerical results and discussions}

This section will solve Eq. (17) by using the finite differential method under two types of initial conditions.

Parameters adopted in simulation are: $\beta=47 \sim 56, \rho_{s}=2400 \mathrm{~kg} / \mathrm{m}^{3}, \rho_{w}=1000 \mathrm{~kg} / \mathrm{m}^{3}, u^{*}=0.04$, $k_{0}=4 \times 10^{-6} \mathrm{~m} / \mathrm{s}, \alpha=1, \kappa=50.0, a=0.08$, time step $\Delta \tau=9 \times 10^{-4}$, step length $\Delta x=0.01$, critical pore pressure $P_{c r}=0.25 \mathrm{MPa}, N=L / \Delta x, L$ is the length of the sand column.

(1) Condition 1: The initial porosity changes continuously. Assume that once a place is choked, the state remains unchanged.

Figure 4 shows that when a place is choked, the porosity below the place increases gradually up to 1.0. A water film is believed to occur there and then expand.

Figure 5 shows the variation of pore water velocity, which increases first because of the high hydraulic gradient and then decreases to zero following the decrease of porosity and permeability. Outside the water film, the velocity changes little, suggesting that there should be discontinuities at the boundaries of water film.

Figure 6 shows the variation of the fine grains eroded from the skeleton. The fine fraction depends on the velocity difference between pore water and grains according to Eqs. (1) and (2). Thus, the value of the fine fraction is small in the water film and places some distance away from the water film, but the value is big near the water film.

Figure 7 shows the distribution of pore pressure. It can be seen that the pore pressure is discontinuous. The peak value occurs at the choked place and causes the upward percolation of pore water. Below the choked place the pore pressure increases suddenly due to the blockage of pore water flow.

Specifically, when the sand column is choked, the velocity of pore water decreases to near zero and a high hydraulic gradient forms at this place due to the very small porosity. Thus the pore water velocity, grain velocity and mass of eroded fine grains tend to be discontinuous. Beneath the choked place, there is a net inflow of pore water because it is difficult for the upward flow to pass through the place, so grains there must move downwards according to Eq. (6). Grains at the above part also move downwards and fill up at the choked place. Then, a water film eventually forms. The pore water beneath the choked place flows upwards while the grains settle down. As this occurs, the water film becomes wider and wider at a expansion rate equal to that of the discontinuity $D=\left[\left(1-\varepsilon^{+}\right) u_{s}^{+}-\left(1-\varepsilon^{-}\right) u_{s}^{-}\right] /\left[\left(1-\varepsilon^{+}\right)-\left(1-\varepsilon^{-}\right)\right]=u_{s}^{-}$, where $u_{s}^{+}, \varepsilon^{+}, u_{s}^{-}$and $\varepsilon^{-}$denote the grain velocity and porosity at the two sides of discontinuity, $u_{s}^{+}=0, \varepsilon^{+}=1$, $u_{s}^{-}$and $\varepsilon^{-}$can be determined by Eq. (20). It can be seen that the expansion rate is the same as the settlement velocity of grains which changes with other parameters of the sand column.

(2) Condition 2: The porosity distribution is the same as that in condition (1), but the choking state may disappear.

Figure 8 shows that when a place is choked, the porosity there increases gradually until a water film develops. However, when the pore pressure is over a critical value, the choking disappears. The pore pressure, pore water velocity and grain velocity become smooth from the discontinuous state. At last, the water film disappears.

Figure 9 shows the velocity of pore water. The velocity tends to be discontinuous after a water film develops and then becomes smooth when the choked place is dredged. 


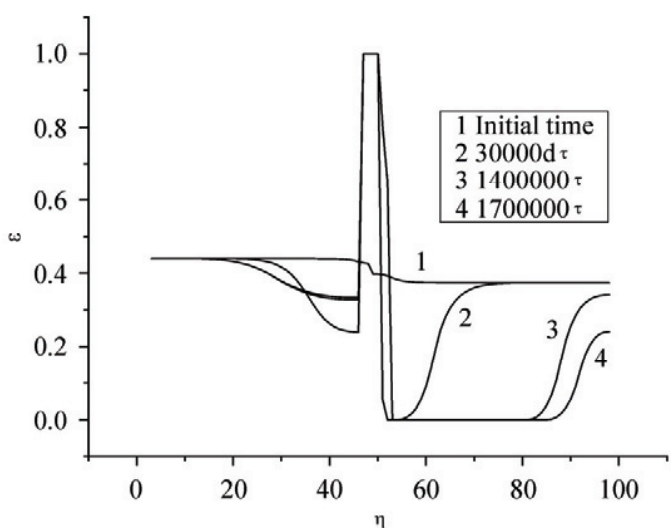

Fig. 4 Development of porosity under assumption 1 (here $N$ equal $L / \Delta x$ )

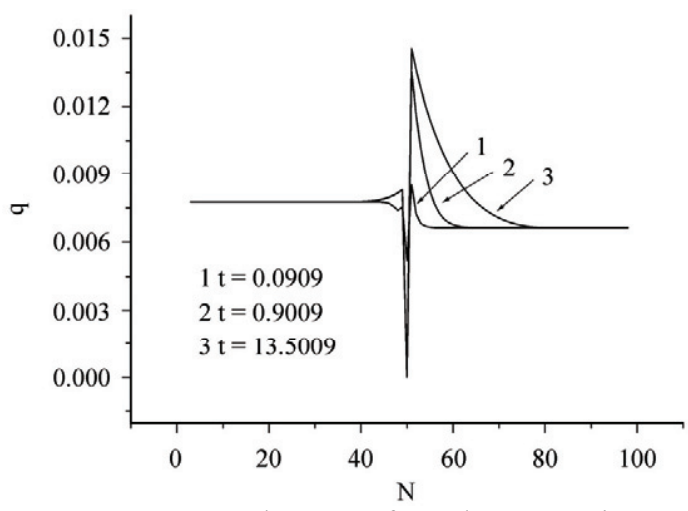

Fig. 6 Development of q under assumption 1

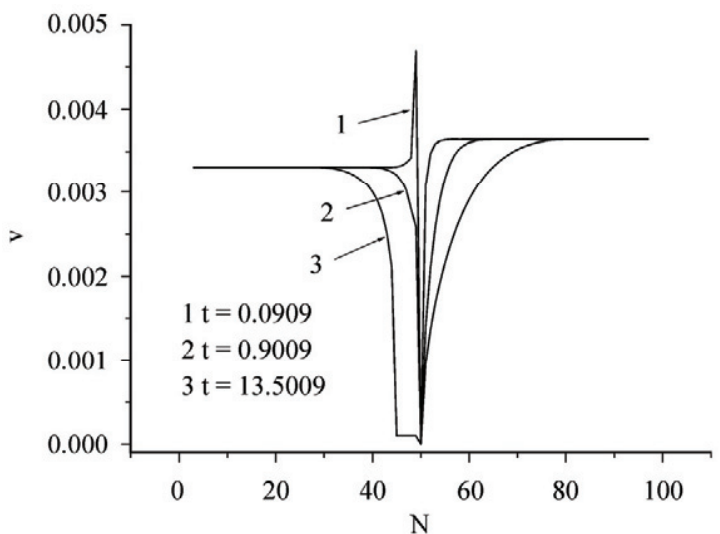

Fig. 5 Development of velocity of pore water under assumption 1

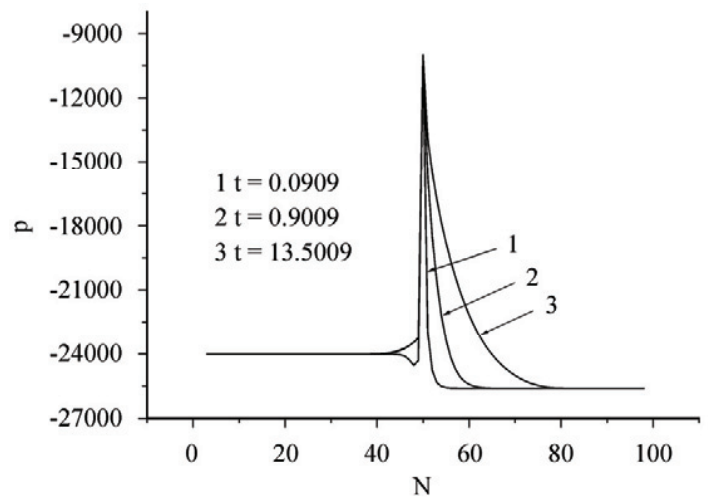

Fig. 7 Development of pore pressure under assumption 1

Figure 10 gives the distribution and variation of the eroded fine grains. The fine fraction decreases sharply in the water film and increases as the water film disappears due to the variation of pore water velocity. After the water film disappears, the value of the fine fraction levels.

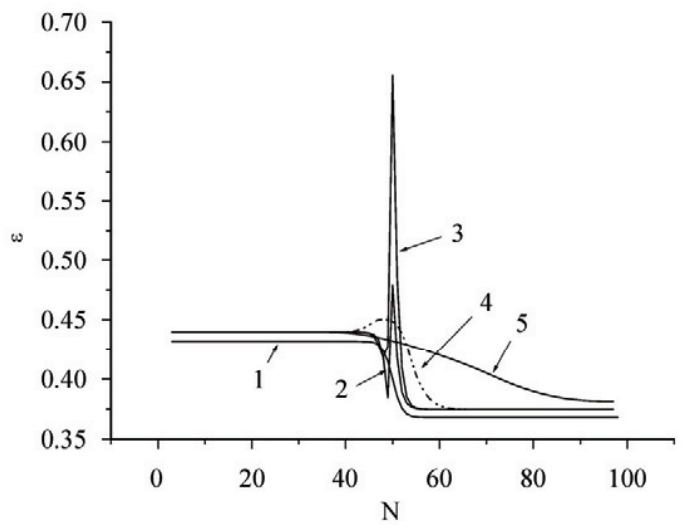

Fig. 8 Development of porosity under assumption 2

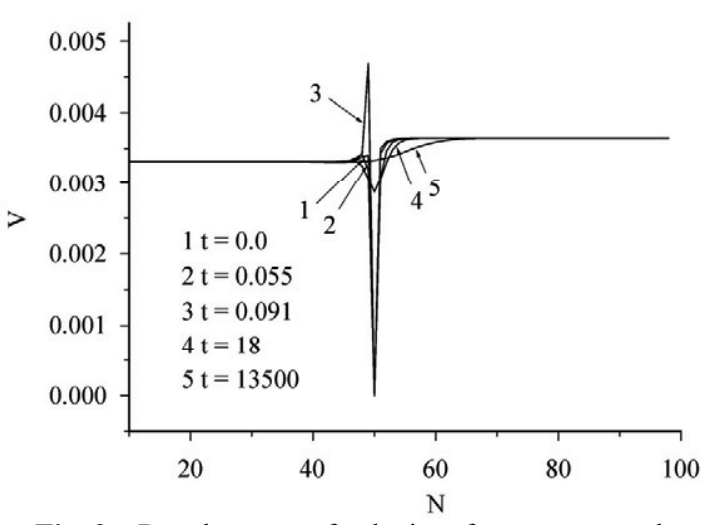

Fig. 9 Development of velocity of pore water under assumption 2

Figure 11 gives the pore pressure, which increases quickly when choked place occurs and decreases with the disappearance of the water film. 


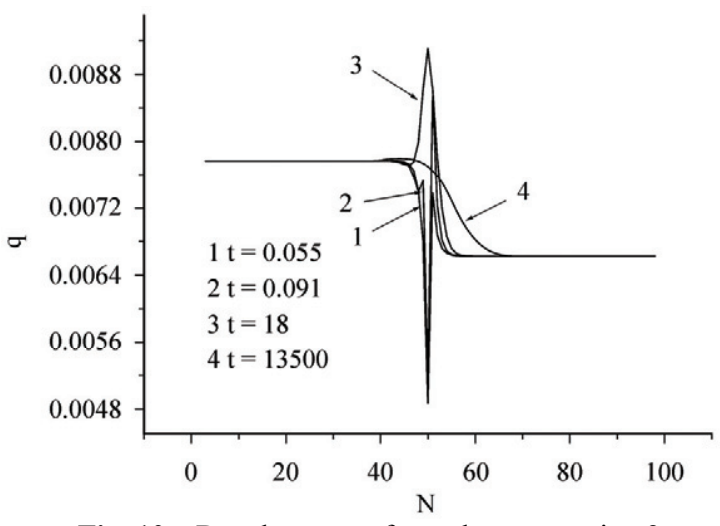

Fig. 10 Development of q under assumption 2

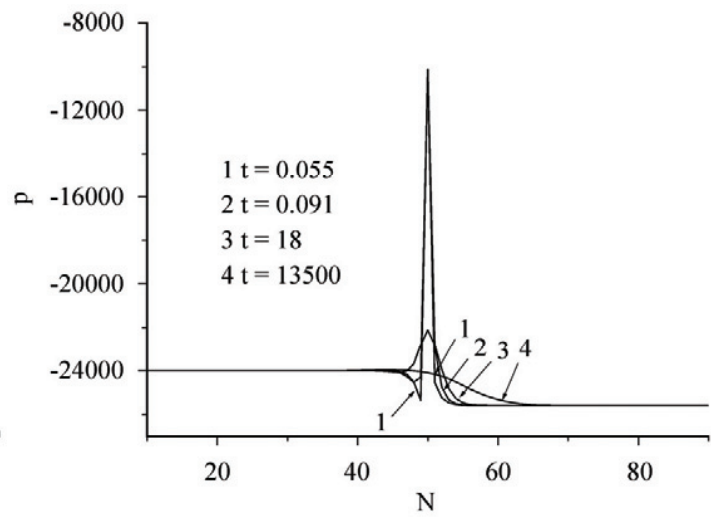

Fig. 11 Development of pore pressure under assumption 2

The water film forms in a similar way in both conditions. But when the pore pressure exceeds a critical value, water can pass through the choked place, the grains above the choked place can settle down again and the pore pressure becomes smooth gradually. Then, the water film disappears.

\section{Comparison with the experimental results}

The numerical results are compared with the experimental data of Kokusho et al. (2002) (Fig.12). In Kokusho's experiment, a saturated loose sand layer of $200 \mathrm{~cm}$ depth sandwiches a seam of non-plastic silt in the middle (96 cm above the bottom). The initial void ratios at the upper, the middle and the lower layers are $0.924,1.5$ and 0.831 , respectively, with corresponding permeability of $0.04 \mathrm{~cm} / \mathrm{s}, 0.00018 \mathrm{~cm} / \mathrm{s}$ and $0.04 \mathrm{~cm} / \mathrm{s}$. The thickness of silt seam is about $4 \mathrm{~mm}$. The saturated sand is in a tube of $13 \mathrm{~cm}$ inner diameter and $211.5 \mathrm{~cm}$ height. The one-dimensional sand layer is instantaneously liquefied by a loading with a steel hammer. The data are adopted in the simulation (Table 1). Figure 12 shows that the two results agree well, under the assumption that the water film begins when the porosity is $10 \%$ higher than the initial porosity.

\section{Conclusions}

Theoretical and numerical analysis have been carried out to investigate the development of water film in saturated sand. The main conclusions can be drawn as follows:

Stable water film may exist in a sand column only if it is liquefied and choked by the eroded fine grains. The non-uniform grain size distribution along the depth of the sand column is an essential precondition for the development of water films. The transport of sand composed of fine grains by percolation tends to aggravate this non-uniformity. Liquefaction is necessary for water films.

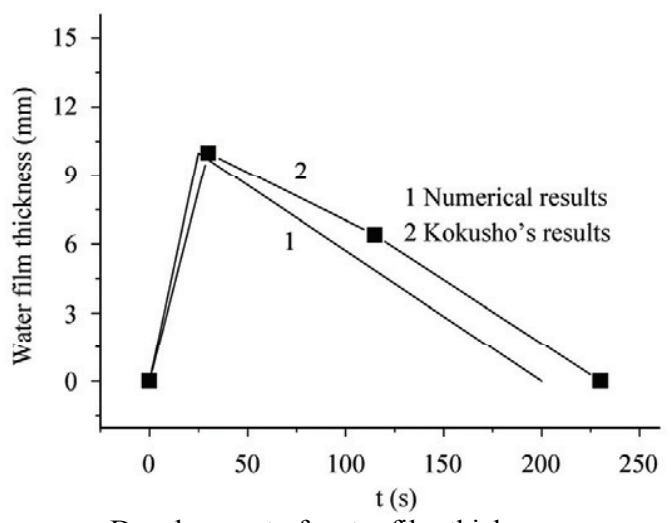

Development of water film thickness

Fig. 12 The comparison of our results with that of Kokusho et al. (2002) 
Table 1 Parameters in Kobusho et al. (2002)

\begin{tabular}{|c|c|c|c|c|c|}
\hline Sand & $\begin{array}{c}\text { Thickness } \\
(\mathrm{cm})\end{array}$ & $\begin{array}{c}\text { Relative density } \\
(\%)\end{array}$ & $\begin{array}{c}\text { Permeability } \\
(\mathrm{cm} / \mathrm{s})\end{array}$ & $\begin{array}{c}\text { Initial } \\
\text { porosity }\end{array}$ & $\begin{array}{c}\text { Maximum of strain } \\
(\%)\end{array}$ \\
\hline Upper layer sand & 103.6 & 14 & 0.04 & 0.48 & 2.4 \\
\hline Sandwich sand & 0.4 & & $1.8 \mathrm{E}-4$ & 0.6 & 2.4 \\
\hline Lower layer sand & 96 & 39 & 0.04 & 0.454 & 0.95 \\
\hline
\end{tabular}

The evolution of the pore water velocity, grain velocity and the amount of fine grains eroded from the skeleton were analyzed. A water film appeared when fine grains eroded from the skeleton and re-deposited downstream in the sand column to cause choking. The coarse grains from above were stopped while they move downwards beneath the choked place. In this way, water films developed. The water film tended to be wider and wider if the choked state was unchanged. Otherwise, the water film disappeared when the pore pressure gradient exceeded a critical value that caused the sand column from choked state to smooth state.

\section{Acknowledgements}

This study was supported by National Basic Research Program of China "Activity characteristics and formation rules of secondary mountain hazard of earthquake" (No. 2008CB425802) and Key Program of Chinese Academy of Sciences (No.KZCX2-YW-302-02). Prof. Li Yong polished the English.

\section{References}

Alekseevskiy N. I., Berkovich K. M., and Chalov R. S. 2008, Sediment transportation and accumulation in rivers. Internaltional Journal of Sediment Research, Vol. 23, No. 21, pp. 93-105.

Bose S. K. and Dey S. 2009, Suspended load in flows on erodible bed. Internaltional Journal of Sediment Research, Vol. 24, No. 3, pp. 315-324.

Cheng C. M., Tan Q. M, Peng F. J. 2000, On the mechanism of the formation of horizontal cracks in a vertical column of saturated sand. ACTA Mechanica Sinica( English Serials), Vol. 17, No. 1, pp. 1-9.

Fazli M., Ghodsian M., and Salehi S. A. A. 2008, Scour and flow field around spur dike in a $90^{\circ}$ bend. Internaltional Journal of Sediment Research, Vol. 23, No. 1, pp. 56-58.

Fiegel, G. L. and Kutter B. L. 1994, Liquefaction mechanism for layered sands. Journal of Geotechnical Engineering, ASCE, Vol. 120, No. 4, pp. 737-755.

Ghodsian M. and Vaghefi M. 2009, Experimental study on scour and flow field in a scour hole around a T-shape spur dike in in a $90^{\circ}$ bend. Internaltional Journal of Sediment Research, Vol. 24, No. 2, pp. 145-158.

Kokusho T. 1999, Water film in liquefied sand and its effect on lateral spread. Jounral of Geotechnical and Geoenvironmental Engineering, No. 10, pp. 817-826.

Kokusho T. and Kojima T. 2002, Mechanism for postliquefaction water film generation in layered sand. Jounral of Geotechnical and Geoenvironmental Engineering, ASCE, Vol. 128, No. 2, pp. 129-137.

Lu X. B., Tan Q. M., Zheng ZM, Yu S. B., and Cui P. 2004, Liquefaction and displacement of saturated sand under vertical vibration loading. Acta Mechanica Sinica, Vol. 20, No. 1, pp. 96-105

Lu X. B., Zheng Z. M., and Wu Y. R. 2006, Formation of water film in saturated sand. Acta Mechanica Sinica, Vol. 22, No. 4, pp. 377-383.

Malvick E. J., Kutter B. L., and Boulanger R. W. 2008, Postshaking shear strain localization in a centrifuge model of a satuarted sand slope. Jounral of Geotechnical and Geoenvironmental Engineering, ASCE, Vol. 134, No. 2, pp. $164-174$.

Malvick E. J., Kutter B. L., Boulanger R. W., and Kulasingam R. 2006, Shear localization due to liquefaction-induced void redistribution in a layered infinite slope. Jounral of Geotechnical and Geoenvironmental Engineering, ASCE, Vol. 132, No. 10, pp. 1293-1303.

Kokusho T. 1999, Water film in liquefied sand and its effect on lateral spread. Jounral of Geotechnical and Geoenvironmental Engineering,Vol. 125, No. 10, pp. 817-826.

Peng F. J., Tan Q. M., and Cheng Z. M. 2001, Laboratory study on water films in saturated sands. Acta Mechanica Sinica, Vol. 16, No. 1, pp. 48-54.

Seed H. B. 1987, Design problems in sand liquefaction. Journal of Geotechical Engineering, ASCE, Vol. 113, No. 8, pp. $827-845$

Scott R. F. 1986, Solidification and consolidation of a liquefied sand column. Soils and Foundations, Vol. 26, No. 4, pp. 23-31.

Yu G. A., Wang Z. Y., Zhang K., Chang T. C., and Liu H. X. 2009, Effect of incoming sediment on the transport rate of bed load in mountain streams. Internaltional Journal of Sediment Research, Vol. 24, No. 3, pp. 260-273.

Wang Z. Y., Wang G. Q., and Huang G. H. 2008, Modeling of state of vegetable and soil erosion over large areas. Internaltional Journal of Sediment Research, Vol. 23, No. 3, pp. 181-196. 
Zhang J. F., Meng X. Y., Yu S. B., Tan Q. M., and Zheng Z. M. 1999, Experimental study on permeability and settlement of saturated sand under impact loading. Acta Mechanica Sinica, Vol. 31, No. 2, pp. 230-237 (in Chinese).

Zheng Z. M., Tan Q. M., and Peng F. J. 2001, On the mechanism of the formation of horizontal cracks in a vertical column of saturated sand. Acta Mechanica Sinica, Vol. 17, No. 1, pp. 1-9. 